increases the chance of disease transmission among these animals. There's also the fact that they have lots of species diversity. More than 1,200 species of bats exist on Earth, accounting for approximately $20 \%$ of all mammalian species. The enormous range of bat species might contribute to why there are so many different bat-borne pathogens. "To say that bats are natural reservoirs of disease would be a simplistic view because there's so much diversity even within bats. Some species of bats can get sick with a certain virus while other species don't," says evolutionary biologist Kevin Olival of the EcoHealth Alliance, a New York-based international organization dedicated to conserving biodiversity.

What's more, as the only mammals capable of flight, bats can travel long distances and, along the way, become exposed to pathogens from different ecological niches much more rapidly than their flightless mammal cousins. Researchers believe that because flight is such an energy-intensive process, it can generate DNA-damaging reactive oxygen species, which trigger an immune response. Having a naturally heightened innate immune response would prime bats to withstand a range of viruses. Furthermore, when bats fly, their body temperature also goes up, so they might thus have more tolerance for pathogens that cause life-threatening fever in other mammals (Emerg. Infect. Dis., 20, 741-745, 2014).

Beyond bats, other examples of reservoir hosts include wild birds, which can harbor avian influenza; rodents, which carry hantaviruses; and nonhuman primates like chimpanzees, which can be infected by simian im munodeficiency virus, a pathogen closely related to HIV. "There are so many natural reservoirs for human pathogens, so there's absolutely a lot we can learn by studying the natural host reservoirs," Olival says "Bats, rodents, and non-human primates together make up about $70 \%$ of all mammalian species, so there's already a huge diversity of potential hosts for viruses."

Each animal has a longstanding evolutionary history with the virus it hosts and has developed ways to cope with it. For example, wild waterfowl, such as mallards and shorebirds, have a cytoplasmic protein called RIG-1 (retinoic acid-inducible gene 1) that senses RNA viruses (Proc. Natl. Acad. Sci.,

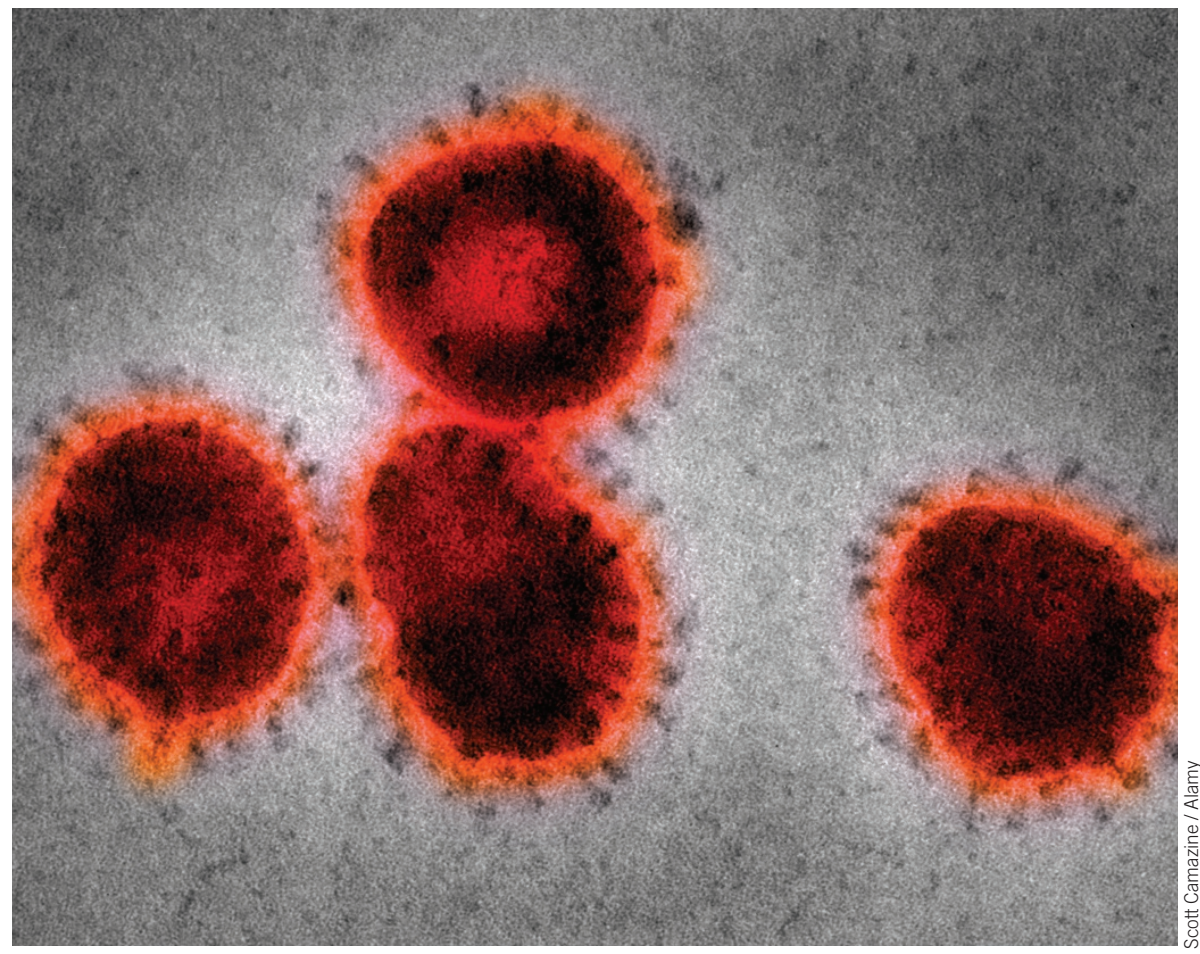

Flight risk: The SARS virus, which resides in bats.

107, 5913-5918, 2010). RIG-I has the ability to turn on a "massive interferon response," in wild waterfowl, allowing these birds to fight avian influenza, according to Katherine Magor, a biologist at the University of Alberta in Canada who studies the immune system of wild birds. Chickens, in contrast, lack RIG-1 and are therefore more susceptible to infection with avian influenza. The adaptive immune response is rapidly switched on in certain rodents, such as deer mice or cotton rats, so that these animals can live comfortably with hantaviruses without appearing sick themselves (Viruses, 6, 1317-1335, 2014).

Amino acid differences in a protein called tripartite motif containing 5 (TRIM-5), which controls viral infection, allows most chimpanzees to tolerate SIV and remain healthy (Nature, 427, 848-853, 2004). "From studying the natural hosts of SIV, we've also learned that chimpanzees can switch off the chronic immune response following virus infection whereas humans don't," says Guido Silvestri, a pathologist at Emory University in Atlanta. "This concept has really shaped the field of HIV drug research: for example, we now know that one of the key factors in preventing infections is having a relatively quiescent immune system, so we want to design interventions that will do the same in humans, as well."

\section{Early clues}

After bats were identified to be the natural viral reservoir for SARS and other infectious diseases, more scientists wanted to understand how bats could tolerate a range of viruses by studying these animals' immune systems. "I started looking at bat immunology in 2008," says Michelle Baker, a bat immunologist at Commonwealth Scientific and Industrial Research Organization (CSIRO), based in Victoria, Australia. "At that point, you could review all the literature on bat immunology in an afternoon. There really wasn't much known, perhaps because nobody was willing to fund that basic research. Now that we know bats can carry all these deadly infectious diseases, people think we needed to study bats."

Baker and her colleagues performed experimental infections of viruses deadly to other animals in bats. Hendra virus, for example, has a case fatality rate between 50 to $100 \%$ in humans (Curr. Opin. Virol., 2, 242-247, 2012). Interestingly, Hendra virus does not cause disease in bats. "You can put a dose of Hendra that's lethal in any other species and bats don't even show a fever," Baker says. "Bats have just become very 
efficient at stopping viruses from replicating and can control them very quickly."

Perplexed by the observation that bats do not fall ill after being infected with a range of viruses, Baker and her colleagues looked more closely at the immune system of bats. They first examined the gene expression profile of Australian black flying foxes, the reservoir for the Hendra and Nipah viruses, and found that these bats have approximately 500 transcribed genes involved in immunity, only a fractionabout a third-of the number of known human immune genes (BMC Genomics, 13, 261, 2012). A comparison of the genome of bats with that of other mammals such as humans, mice and chimpanzees revealed that genes associated with the innate immune response, such as those that encode interferon receptor 1, interleukin-18, interferon-gamma, and toll-like receptor were positively selected for in bats compared to humans and mice (Science, 339, 456-460, 2013). Many of these genes, according to Wang, also a co-author on the Science study, could help explain why bats have a more effective immune system that allows them to to prevent these viruses from replicating so that the viruses don't cause disease in the animal.

These studies led Peng Zhou, a coauthor on the 2013 Science study and, at the time, a postdoctoral fellow in Baker's lab at CSIRO, to look more closely at interferon production in bats. Now Zhou continues to study bat immunology at Duke-NUS Medical School with Wang. Interferons are signaling proteins produced and secreted by cells in response to infection that can activate the adaptive immune response. Zhou found that bats were making interferon-alpha (IFN- $\alpha$ ) RNA even when they were not infected with any detectable virus. " [IFN- $\alpha$ RNA] is almost undetectable in other mammals that are not stimulated with a virus, whereas in bats, IFN- $\alpha$ RNA is already very high even without viral challenge," Baker says. What is considered to be a normal baseline IFN- $\alpha$ level for bats would be toxic to humans or mice, potentially causing flu-like symptoms or organ damage. Unpublished data from Baker's lab showed that IFN- $\alpha$ RNA levels in bats hardly increased even when they were challenged with a range of viruses like Hendra, another bat virus called Pulau virus, and a murine virus called Sendai. The high baseline levels of IFN- $\alpha$ RNA, according to Zhou, could explain why bats can coexist with viruses without appearing sick. "However, how bats can tolerate those consistently high levels of IFN- $\alpha$ remains widely unknown," Zhou says.

Zhou has continued to examine why IFN- $\alpha$ messenger RNA is higher in bats

than in other animals. Unpublished data from Zhou and Baker suggests that sequence differences in the upstream, promoter region of the gene that encodes IFN- $\alpha$ could explain why the message is much higher in bats compared with other mammals.

\section{Looking forward}

Since the SARS outbreak in 2003, the world has witnessed two other major outbreaks of diseases in which bats are thought to be natural reservoirs: MERS in 2012 and Ebola in 2014 (Emerg. Infect. Dis., 19, 1819-1823, 2013; Emerg. Infect. Dis., 19, 270-273, 2013). The emergence of these diseases creates a greater imperative for scientists to better understand how the animals that are natural reservoirs of these pathogens respond to ongoing infection, and to harness this information for drug development.

"The question about how bats cope with disease is important," says Christopher Basler, a microbiologist at the Mount Sinai School of Medicine in New York. "It's a big question, and a lot of people are interested in it. For now, I would look at the data carefully: which bats are being studied? What cell types are examined?"

"We're just beginning to learn what's special about bats," says Zheng-li

"We need to build
our own toolbox for
now as there are no
reagents to study bat
immunology."

Shi, a virologist at Wuhan

Institute of Virology in Wuhan, China. She was on the team of researchers who identified bats as the natural reservoir of SARS in 2005. "The aim that we want to reach, of bringing these lessons from bats into humans, is far away from what we have done so far."

Because bat immunology is still a fairly nascent field, there is a dearth of available reagents for research. More than twenty bat cell lines have been generated to be used for research, compared to nearly 4,000 human cell lines available through the American Type Culture Collection (ATCC), the world's cell culture repository based in Virginia (PLoS ONE, 4, e8266, 2009). Developing antibodies for bat-specific proteins has also been challenging: for example, an antibody that specifically recognizes bat IFN- $\alpha$ does not yet exist. "All we are measuring right now is RNA levels because we don't have the tools to go deeper," Wang says. "We need to build our own toolbox for now as there are no reagents to study bat immunology." Furthermore, studying bats in the wild can be challenging; many bat researchers are starting to generate their own bat colonies and facilities for animal experiments.

the disease. they harbor.
To prove that IFN- $\alpha$ RNA is being translated and carrying out its expected functions, Zhou had to utilize overexpression systems. Unpublished data from Zhou suggests that the overexpressed IFN- $\alpha-$ encoding genes are indeed being translated into proteins that can turn on downstream interferon-stimulated genes.

\section{Past predictors}

The deadly toll of SARS underscores the need for researchers to study natural reservoirs of disease. Unfortunately for Jianlun Liu, he did not escape unscathed: on 22 February 2003, a day after Liu checked in to the hotel, he sought urgent care at the nearby Kwong Wah Hospital. When he was admitted to the intensive care unit, Liu mentioned that he had treated patients with atypical pneumonia and warned the medical staff he may have contracted a "very virulent disease." He passed away on 4 March 2003. When the WHO announced that the virus was finally contained on 5 July 2003, more than 8,000 cases and more than 800 deaths were reported internationally. A year after the SARS outbreak, the Metropole Hotel in Hong Kong renumbered the room where Liu had stayed in an attempt to erase all memories of

Although it's difficult to predict when and what the next epidemic will be, evolutionary biologists and virologists already have plenty of interesting questions from studying the immune systems of natural hosts to keep them busy. Bats evolved to their present form approximately 50 million years ago, and they are likely to have a longstanding co-evolutionary history with the viruses

"People talk about the work that we do as an insurance policy: we've got to be on top of what's happening with the viruses that are carried by bats and understand what goes on in a bat's immune system to possibly predict a spillover event into another species," Baker says. The next virus could cause an epidemic like SARS, but Olival says that clues from bats can help us be "more proactive, rather than reactive, to the next 'big one."

\section{Corrected after print 28 April 2016}

Wudan Yan is a freelance science journalist and a former news intern at Nature Medicine. 


\section{Correction}

In the version of the article "Going batty: studying natural reservoirs to inform drug development" initially published, (Nat. Med. 21, 831-833, 2015), there were editorial comments included from an earlier edited version of the story. The error has been corrected in the HTML and PDF versions of the article. 